\title{
Active (p)CrkL is overexpressed in human malignancies: Potential role as a surrogate parameter for therapeutic tyrosine kinase inhibition
}

\author{
CHRISTIAN F. SINGER ${ }^{1 *}$, GERNOT HUDELIST ${ }^{4 *}$, WOLFGANG LAMM $^{1}$, RUTH MUELLER $^{1}$, \\ CLAUDIA HANDL $^{3}$, ERNST KUBISTA ${ }^{1}$ and KLAUS CZERWENKA ${ }^{2}$ \\ ${ }^{1}$ Division of Senology and Ludwig Boltzmann-Institute of Clinical Experimental Oncology and ${ }^{2}$ Department of \\ Gynecopathology, University of Vienna, Vienna; ${ }^{3}$ Novartis Austria GmbH, Vienna; \\ ${ }^{4}$ Department of OB/GYN, LKH Villach, Villach, Austria
}

Received June 7, 2005; Accepted July 25, 2005

\begin{abstract}
CrkL is a nuclear adaptor and transcriptional activator in Bcr-Abl expressing cells and constitutes the major tyrosine phosphorylated protein in CML, but the expression and biological function of CrkL in other malignancies is largely unknown. Using immunohistochemistry, we have analyzed the protein expression of activated (p)CrkL in normal and malignant tissues. We then treated K562 leukemia cells with imatinib to analyze the effect of tyrosine kinase inhibition on CrkL activation. pCrkL expression was predominantly epithelial and detected in the majority of non-malignant prostate (79\%), $49 \%$ of colon biopsies, $36 \%$ of skin biopsies, and $41 \%$ of samples obtained from normal brain. Protein expression was, however, considerably less frequent in normal breast (18\%), lung (16\%) and ovarian (12\%) tissues. In contrast to their corresponding benign tissues, pCrkL expression was significantly more common in breast cancer samples $(49 \%, \mathrm{p}<0.0001$; Fisher's exact test), lung carcinomas $(55 \%, \mathrm{p}=0.0002)$, lymphatic tissues $(80 \%$ vs. $10 \%, \mathrm{p}=0.012)$, skin cancer $(67 \%, \mathrm{p}=0.020)$, ovarian malignomas $(50 \%, \mathrm{p}<0.0001)$ and colon carcinomas $(63 \%, \mathrm{p}<0.03)$. By contrast, activated CrkL was significantly less frequent in prostate carcinoma samples when compared to corresponding non-malignant prostatic tissues (14\% vs. $79 \%, \mathrm{p}<0.0001)$. pCrkL expression was abrogated in K562 cells with the addition of the tyrosine kinase inhibitor imatinib, which indicates that phosphorylation of CrkL is mediated through targets of therapeutic TK inhibition. We hypothesize that pCrkL is selectively up-regulated in a number of malignant tumor entities and involved in malignant
\end{abstract}

Correspondence to: Dr Christian F. Singer, Department of Obstetrics and Gynecology, Division of Senology, Vienna Medical University, Waehringer Guertel 18-20, 1090 Vienna, Austria

E-mail: christian.singer@meduniwien.ac.at

\section{${ }^{*}$ Contributed equally}

Key words: pCrkL, imatinib, tyrosine kinase inhibitor, malignancies transformation. We further suggest that $\mathrm{pCrkL}$ might serve as a potential surrogate parameter for the efficacy of therapeutic TK inhibition.

\section{Introduction}

While some proteins directly interact with their molecular targets, others depend on interactions that are mediated by a type of molecule known as adaptor proteins. Adaptor proteins usually do not have catalytic properties themselves, but function by bringing two or more proteins together through the utilization of one or more distinct binding domains. One such adaptor protein is Crk, which has initially been found to be the oncogenic product of the avian sarcoma virus CT10, and leads to an increase in tyrosine-phosphorylated proteins in the absence of an apparent enzymatic domain (hence the term Crk for chicken tumor virus CT10 regulator of kinase) (1). Other members of the Crk family include CrkI, CrkII, CrkIII and Crk-like (CrkL), which all share a high degree of homology in their overall structure (2).

The protein sequence of CrkL contains one Src homology 2 (SH2) and two Src homology 3 (SH3) domains and exhibits a $60 \%$ homology to CrkII, its closest family member (2). SH2 and $\mathrm{SH} 3$ are small protein modules found in a number of signaling proteins that mediate protein-protein interactions in signal transduction pathways activated by protein tyrosine kinases $(3,4)$. Selective formation of multi-protein complexes by Crk family proteins depends on specific motifs recognized by their $\mathrm{SH} 2$ and $\mathrm{SH} 3$ domains. In the case of CrkL, the $\mathrm{SH} 2$ module binds to short phosphotyrosine containing sequences in the intracellular domain of growth factor receptors such as PDGFR or IGF1-R, while the two SH3 domains interact with specific target proteins containing proline-rich sequences. The resulting multi-subunit complex then leads to further signal transduction and modification (5).

Members of the Crk family adaptor proteins are widely expressed in a number of tissues and mediate the formation of signal transduction complexes upon a variety of extracellular stimuli. Crk family proteins appear to play a key role in mediating the action of human oncogenes like the leukemia-inducing Bcr-Abl protein, and CrkL has been 
identified as the major tyrosine-phosphorylated protein in neutrophils from patients with CML (6). CrkL has also been found to be the linking protein between Bcr-Abl and Stat signaling (7). This functional quality, which is thought to be critical in those cases of CML in which the reciprocal translocation between the arms of chromosomes 9 and 22, leads to the generation of a Bcr-Abl fusion protein with a profoundly elevated protein tyrosine kinase (TK) activity (8). Other biological features of Crk-mediated signal transduction cascades include the integrin-mediated cell adhesion in hematopoietic cell lines and epithelial-mesenchymal-like transitions in the human breast cancer cell line T47D $(9,10)$. Crk family-mediated phenotypical changes also involve alterations in cell migration and immune cell responses $(11,12)$.

Activation of Crk family members, mediated by transphosphorylation, is usually the result of either the ligandmediated activation of a transmembrane receptor molecule, or the consequence of a constitutitively activated membranebound TK. The abrogation of TK activity by synthetic TK inhibitors (TKIs) such as imatinib $\left(\right.$ Glivec $\left.^{\circledR}\right)$ consequently leads to the inactivation of downstream signaling in selected diseases associated with constitutively activated TKs. We therefore performed pCrkL immunohistochemistry to evaluate the presence of activated TKs in a series of human malignant tumors. We also investigated pCrkL expression in the CrkL expressing cell line $\mathrm{K} 562$ in response to the TK inhibitor inatinib in order to exemplify the effect of TK inhibition on the activation state of CrkL.

\section{Materials and methods}

Tissue arrays. Fourth generation tissue arrays (TARP-4) were purchased from the National Cancer Institute Tissue Array Program. Each array contained 500 tissue samples from biopsies obtained from malignant brain tumors (25 samples), malignant breast tumors (75 samples), malignant colon tumors (75 samples), lung cancer (75 samples), lymphomas (50 samples), malignant melanomas (25 samples), ovarian cancer (50 samples), prostate cancer ( 75 samples) and corresponding normal tissues (see Table I). A detailed listing of parameters can be found for each individual tumor biopsy at http://ccr.cancer.gov/tech_initiatives/tarp. Tissue arrays containing multiple samples from normal multiple tissues (\#BN00012), brain (\#GL801), breast (\#BN08013), colon (\#BN05021), lung (\#BN04011), skin (\#SK801), ovary (\#OV801) and prostate (\#BC19111) were obtained from Biomax (Biomax Inc., Rockville, MD).

Immunohistochemistry. Tissue arrays were deparaffinized with 3 washes in xylene for 5 min each, followed by 2 washes in $100 \%$ ethanol for $10 \mathrm{~min}$ each. The slides were then incubated in $95 \%$ ethanol for $10 \mathrm{~min}$ and washed twice in $\mathrm{dH}_{2} \mathrm{O}$ for $5 \mathrm{~min}$. For antigen unmasking, sections were microwaved in $10 \mathrm{mM}$ sodium citrate buffer ( $\mathrm{pH}$ 6.0) for $1 \mathrm{~min}$. After 3 washes in $\mathrm{dH}_{2} \mathrm{O}$ for 5 min each, sections were incubated in $1 \%$ hydrogen peroxide for $10 \mathrm{~min}$ to quench endogenous peroxidase activity, then washed 3 times in $\mathrm{dH}_{2} \mathrm{O}$ for $5 \mathrm{~min}$ and rinsed in PBS for another 5 min. The sections were then blocked with goat serum (ImmunoCruz ${ }^{\mathrm{TM}}$ Staining System; Santa Cruz Biotechnology, Santa Cruz, CA, USA) for $1 \mathrm{~h}$
Table I. pCrkL protein expression in normal human tissues.

\begin{tabular}{llcr}
\hline Tissue type & No. analyzed & $\begin{array}{c}\text { pCrkL } \\
\text { expression }\end{array}$ & $\%$ \\
\hline Brain & $\mathrm{n}=22$ & 9 & $41 \%$ \\
& Normal tissue $(\mathrm{n}=17)$ & 8 & $47 \%$ \\
& Benign tumors $(\mathrm{n}=5)$ & 1 & $20 \%$ \\
Breast & Normal tissue $(\mathrm{n}=73)$ & 13 & $18 \%$ \\
Colon & Normal tissue $(\mathrm{n}=71)$ & 35 & $49 \%$ \\
Upper GI tract & Normal tissue $(\mathrm{n}=4)$ & 1 & $25 \%$ \\
Lung & Normal tissue $(\mathrm{n}=28)$ & 4 & $16 \%$ \\
Lymph node & Normal tissue $(\mathrm{n}=5)$ & 1 & $20 \%$ \\
Skin & Normal tissue $(\mathrm{n}=14)$ & 5 & $36 \%$ \\
Ovary & Normal tissue $(\mathrm{n}=33)$ & 4 & $12 \%$ \\
Endometrium & Normal tissue $(\mathrm{n}=2)$ & 1 & $50 \%$ \\
Prostate & $\mathrm{n}=24$ & 19 & $79 \%$ \\
& Normal tissue $(\mathrm{n}=18)$ & 13 & $72 \%$ \\
& Benign hyperplasia $(\mathrm{n}=6)$ & 6 & $100 \%$ \\
Glandular tissue & Normal tissue $(\mathrm{n}=6)$ & 4 & $67 \%$ \\
Liver & Normal tissue $(\mathrm{n}=3)$ & 3 & $100 \%$ \\
Bladder & Normal tissue $(\mathrm{n}=2)$ & 1 & $50 \%$ \\
\hline
\end{tabular}

at room temperature. After consecutive PBS washes, the slides were incubated overnight at $4^{\circ} \mathrm{C}$ with the phosphoCrkL (Tyr 207) antibody (Cell Signaling Technology Inc., Beverly, MA, USA) at a dilution of 1:50. Negative controls were performed on all tissue arrays by replacing primary antibodies with diluted isotype immunoglobines (ImmunoCruz Staining System, Santa Cruz Biotechnology). Slides were then washed 3 times with PBS, and incubated with the biotinylated secondary antibody provided in the detection kit and horseradish peroxidase (HRP) agent for $30 \mathrm{~min}$, then incubated with the substrate-chromogen 3,3'-diaminobenzidine for $5 \mathrm{~min}$. All sections were counterstained with hematoxylin for $10 \mathrm{sec}$, dehydrated in $95 \%$ and $100 \%$ ethanol, and mounted on coverslips.

Cell line and cell culture conditions. K562 cells were purchased from the American Type Culture Collection (ATCC; Manassas, VA, USA) and grown in Dulbecco's modified Eagle's medium (DMEM; Gibco, Vienna, Austria), supplemented with $2 \mathrm{mM}$ GlutaMax (Gibco $100 \mu \mathrm{g} / \mathrm{ml}$ streptomycin, $100 \mathrm{U} / \mathrm{ml}$ penicillin and $10 \%$ fetal calf serum (FCS; PAA Laboratories, Linz, Austria). Imatinib mesylate (STI-571; Glivec) was kindly provided by Novartis Pharmaceuticals (Basel, Switzerland) and prepared as a $10 \mathrm{mmol} / \mathrm{l}$ stock solution in sterile dimethylsulphoxide (DMSO; Sigma, St. Louis, MO, USA). Upon subconfluency, cells were washed 3 times in PBS, and FCS-containing medium was replaced with FCS-free IMDM with either $500 \mathrm{nM}$ imatinib mesylate or $0.1 \%$ DMSO containing medium alone. Cells were then maintained for another $24 \mathrm{~h}$, then harvested and subjected to a cytospin before immunocytochemistry was performed.

Western blot analysis. Western blotting was used to confirm antibody specificity. After washing twice with PBS, a confluent 
Table II. pCrkL protein expression in human malignancies.

\begin{tabular}{|c|c|c|c|}
\hline Tumor type & Histology & pCrkL expression & $\%$ \\
\hline \multirow[t]{3}{*}{ Malignant brain tumors } & $\mathrm{n}=84$ & 47 & $56 \%$ \\
\hline & Glioblastoma multiforme $(n=24)$ & 18 & $75 \%$ \\
\hline & Astrocytoma $(\mathrm{n}=60)$ & 29 & $48 \%$ \\
\hline \multirow[t]{5}{*}{ Breast cancer } & $\mathrm{n}=70$ & 34 & $49 \%$ \\
\hline & Invasive ductal $(\mathrm{n}=57)$ & 28 & $49 \%$ \\
\hline & Invasive lobular (n=9) & 4 & $44 \%$ \\
\hline & DCIS $(n=1)$ & 0 & $0 \%$ \\
\hline & Unknown $(\mathrm{n}=3)$ & 2 & $67 \%$ \\
\hline \multirow[t]{3}{*}{ Colon cancer } & $\mathrm{n}=70$ & 44 & $63 \%$ \\
\hline & Adenocarcinoma (n=64) & 43 & $67 \%$ \\
\hline & Mucinous (n=6) & 1 & $17 \%$ \\
\hline \multirow[t]{6}{*}{ Lung cancer } & $\mathrm{n}=71$ & 39 & $55 \%$ \\
\hline & Adenocarcinoma $(\mathrm{n}=25)$ & 18 & $72 \%$ \\
\hline & Squamous (n=32) & 14 & $44 \%$ \\
\hline & Mucinous (n=2) & 2 & $100 \%$ \\
\hline & Bronchoalveolar $(\mathrm{n}=2)$ & 1 & $50 \%$ \\
\hline & Unknown $(\mathrm{n}=10)$ & 4 & $40 \%$ \\
\hline \multirow[t]{3}{*}{ Lymphoma } & $\mathrm{n}=50$ & 40 & $80 \%$ \\
\hline & Hodgkin's lymphoma (n=5) & 4 & $80 \%$ \\
\hline & Other $(n=45)$ & 36 & $80 \%$ \\
\hline \multirow[t]{4}{*}{ Skin cancer } & $\mathrm{n}=89$ & 60 & $67 \%$ \\
\hline & Melanoma (n=35) & 20 & $57 \%$ \\
\hline & Squamous cell cancer $(n=40)$ & 30 & $75 \%$ \\
\hline & Basal cell cancer $(n=14)$ & 10 & $71 \%$ \\
\hline \multirow[t]{4}{*}{ Ovarian cancer } & $\mathrm{n}=86$ & 43 & $50 \%$ \\
\hline & Serous $(n=69)$ & 35 & $51 \%$ \\
\hline & Endometrioid $(\mathrm{n}=5)$ & 2 & $40 \%$ \\
\hline & Mucinous or clear cell $(n=12)$ & 6 & $50 \%$ \\
\hline \multirow[t]{2}{*}{ Prostate cancer } & $\mathrm{n}=73$ & 10 & $14 \%$ \\
\hline & Adenocarcinoma (n=73) & 10 & $14 \%$ \\
\hline
\end{tabular}

$75 \mathrm{~cm}^{2}$ cell culture flask was trypsinized for $3 \mathrm{~min}$. Detached cells were then suspended in $0.5 \mathrm{ml}$ Laemmli buffer and boiled for $5 \mathrm{~min}$, before aliquots of $10 \mu \mathrm{l}$ were loaded onto a $7.5 \%$ SDS polyacrylamide gel (PAGE) and separated by employing a constant voltage of $200 \mathrm{~V}$ for $30 \mathrm{~min}$. After transfering to nitrocellulose membranes (Invitrogen, Carlsbad, CA, USA), the protein extracts were either subjected to a pre-treatment with 3 washing steps in $\mathrm{ddH}_{2} \mathrm{O}$ and subsequent overnight exposure to $5 \%$ (vol/vol) calf intestine phosphatase (CIP; Amersham Bioscience, Uppsala, Sweden) at $37^{\circ} \mathrm{C}$, or directly incubated with the phospho-CrkL (Tyr 207) antibody (Cell Signaling Technology), or CrkL antibody (Cell Signaling Technology) at a dilution of 1:1000 for $8 \mathrm{~h}$ at room temperature. A rainbow molecular size marker (Amersham Biosciences) was used for protein size determination.

Immunostaining quantification and statistical analysis. A semiquantitative scoring system (immunoreactive score; IRS) was used to allow for a reproducible evaluation of immunohistochemically stained tissue sections (13). The IRS with staining intensities of negative, weak, moderate and strong (multiplied with the percentage of positive cells or nuclei) ranged from 0 and 12. A range of 0 to 2 points was considered to be negative, weak staining ( 3 to 5 points) was defined as $1+$, moderate staining ( 6 to 8 points) as $2+$ and strong staining ( 9 to 12 points) as $3+$ cytoplasmatic staining. The SAS 8.2 TS2M0 Win software package was used to perform statistical analyses and a two-sided $\mathrm{p}<0.05$ was considered statistically significant.

\section{Results}

Specificity of the phospho-specific (p-Tyr 207) CrkL antibody. The specificity of the pCrkL antibody was assessed by Western blotting of K562 protein extracts (Fig. 1). K562 cells derive from a human pluri-potent chronic myeloic leukemia 

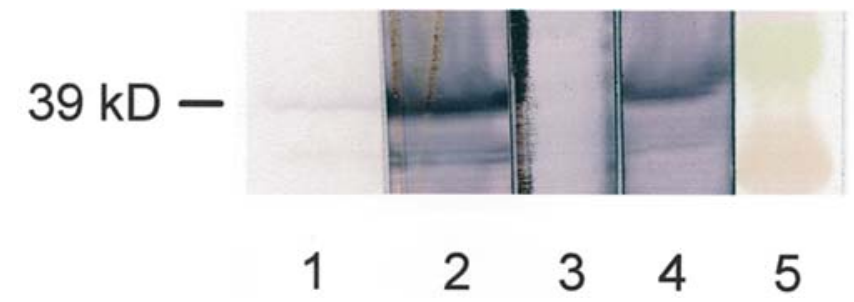

Figure 1. The pCrkL Ab specifically detects the Tyr 207-phosphorylated form of CrkL with Western blot analysis of K562 cell protein extracts treated with or without CIP after membrane transfer. Total cellular protein extract incubated with the pCrkL Ab (lane 1) and the CrkL Ab (lane 2) without prior exposure to CIP. Total cellular protein extract incubated with pCrkL Ab after prior dephosphorylation with CIP (lane 3). Total cellular protein extract incubated with $\mathrm{CrkL} \mathrm{Ab}$ after prior dephosphorylation with CIP (lane 4). Whole-range rainbow protein size marker (lane 5).
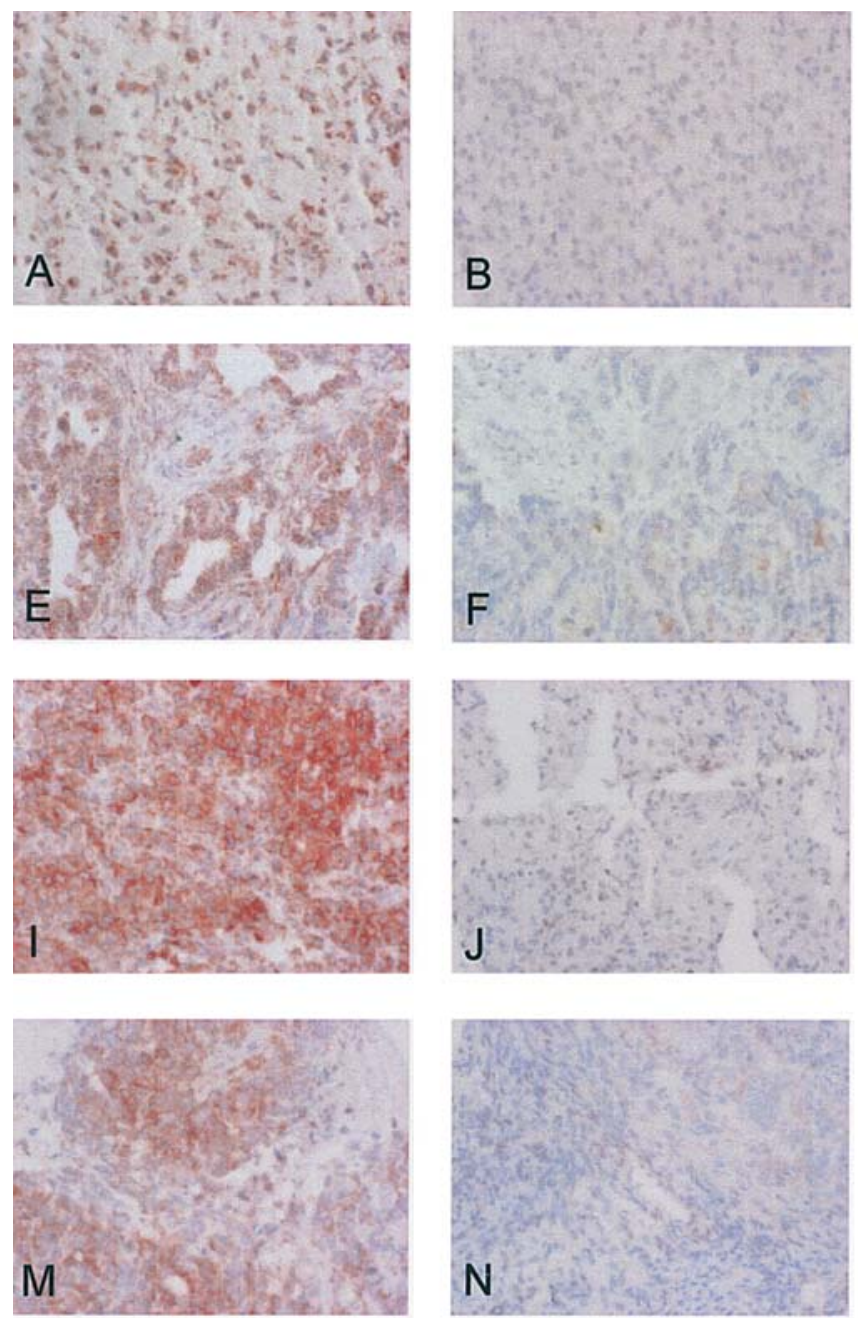

cell line in which CrkL is constitutively activated due to a chimeric bcr/c-abl transcript. When cell extracts from K562 cells were subjected to immunoblotting and incubated with the anti-pCrkL antibody as described in Materials and methods, a single band at $39 \mathrm{kDa}$ was visible (lane 1). A strong band of comparable molecular size was detected after incubation with an anti-CrkL antibody (lane 2). When the cell extracts were subjected to overnight treatment with CIP prior to immunodetection with $\mathrm{pCrkL}$, which results in a dephosphorylation of phospho-proteins, the band at the expected size was no longer detectable (lane 3). However, when protein extracts were dephosphorylated with CIP, but then incubated with the CrkL antibody (which detects CrkL protein regardless of its phosphorylation state), a distinct band at $39 \mathrm{kDa}$ was again seen (lane 4).
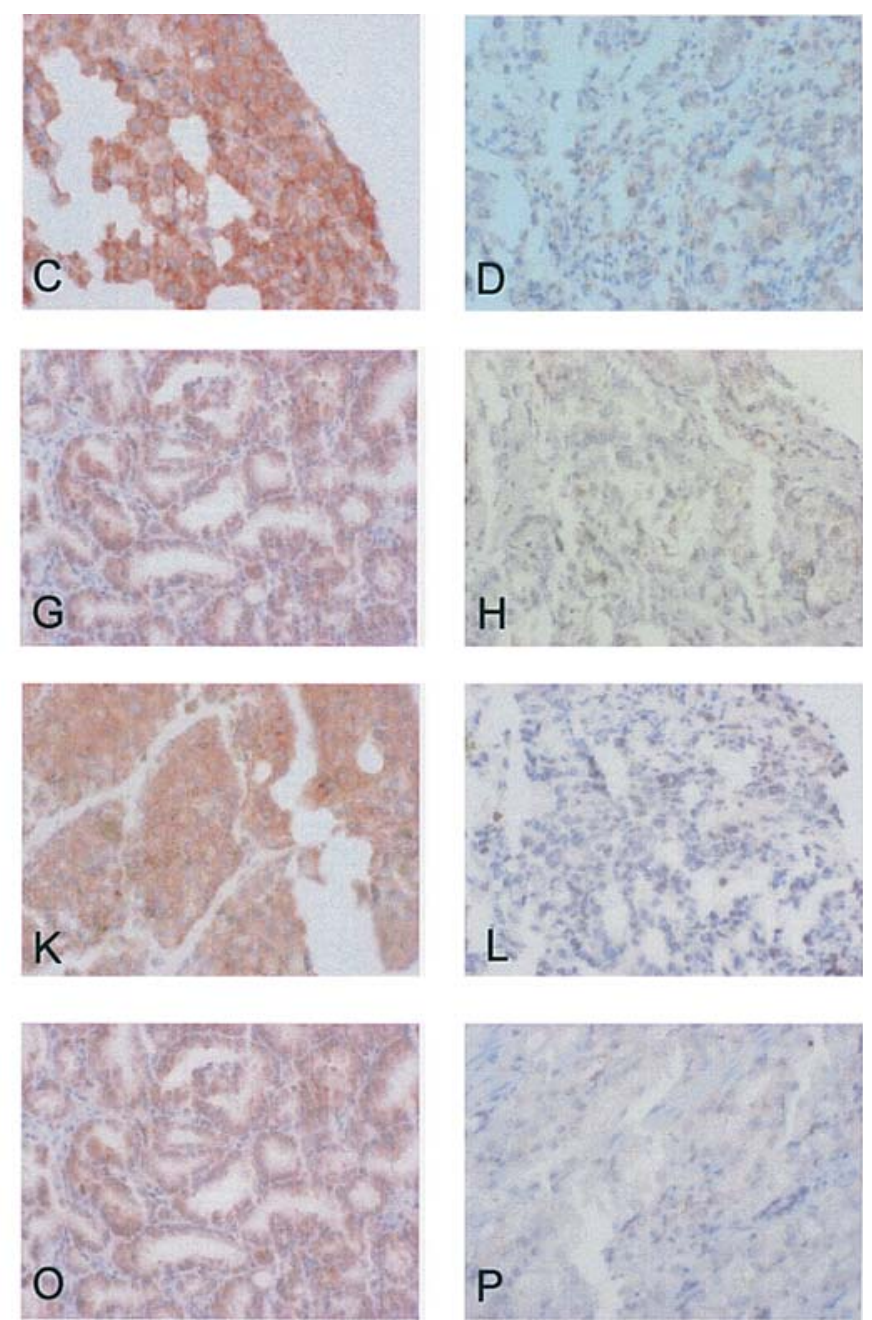

Figure 2. Immunohistochemical analysis of pCrkL staining in human malignancies. Representative cases of positive and negative pCrkL staining are shown here of malignant brain tumors ( $\mathrm{A}$ and $\mathrm{B}$ ), breast cancer (C and D), colon cancer (E and F) lung cancer (G and H), lymphoma (I and J), melanoma (K and I) ovarian cancer $(\mathrm{L}$ an $\mathrm{M})$ and prostate cancer $(\mathrm{N}$ and $\mathrm{O})$. Staining is confined to malignant cells and absent in surrounding stroma.
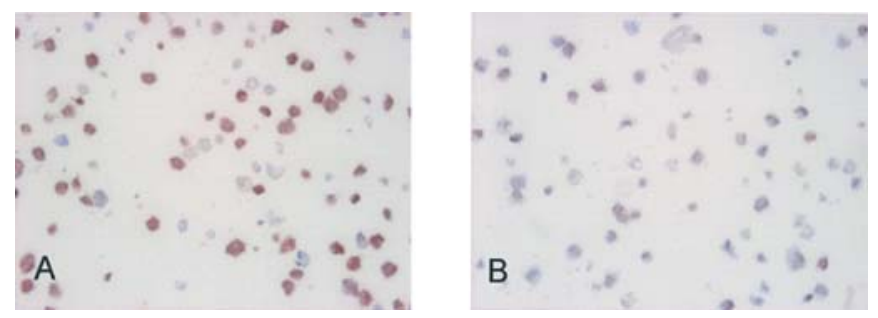

Figure 3. pCrkL expression in K562 cytospins in the absence (A), and lack of pCrkL protein expression in the presence of $500 \mathrm{nM}$ STI 571 (B), treated as described in Materials and methods. 
pCrkL protein expression in normal human tissues. Immunohistochemical pCrkL protein expression in normal human tissues is shown in Table I. Some 9 of 22 non-malignant brain samples (41\%) expressed active (p)CrkL, while normal colon samples exhibited pCrkL positivity in 35 of 71 cases (49\%). In non-malignant prostate tissue, pCrkL positivity was even detected in 19 of 24 samples investigated (79\%). Interestingly, while microscopically normal prostate tissue expressed in 13/18 cases (72\%), all 6 biopsies obtained from benign prostate hyperplasias were positive for pCrkL (100\%). In most other tumor entities, however, the presence of the activated form of CrkL was considerably less frequent. In normal breast tissue, pCrkL was observed in only 13 of the 73 samples investigated (18\%). Similarly, only 4 of 28 biopsies were pCrkL positive (16\%) in lung tissue, and normal ovarian tissue exhibited pCrkL staining in 4 of 33 samples (12\%).

In addition, we also found pCrkL in all samples obtained from the upper gastrointestinal tract (stomach), 3 of 3 samples obtained from normal liver, 4 of 6 specimens of glandular tissue, 1 of 2 bladder samples, 1 of 2 endometrial samples and 1 of 5 biopsies (20\%) obtained from normal lymph nodes.

pCrkL expression in human malignancies. pCrkL protein expression in human malignancies is summarized in Table II, and representative cases are shown in Fig. 2. pCrkL was detected in 47 of 84 evaluable malignant brain tumors (56\%) with 18 of 24 glioblastoma multiforme $(75 \%)$ and 29 of 60 astrocytoma (48\%) being pCrkL-positive. If present, low levels of protein (+) were unvariably expressed throughout the malignant tissue (Fig. 2A). No significant difference in pCrkL expression was found when malignant and nonmalignant tissues were compared ( $\mathrm{p}=0.087$; Fisher's exact test). In malignant breast tissue, however, pCrkL expression was significantly more common than in normal breast tissue ( $p<0.0001$; Fisher's exact test), with $49 \%$ of 70 samples being pCrkL-positive. Staining intensities varied widely, ranging from weak $(28 \%)$ via intermediate $(16 \%)$ to strong $(5 \%)$. If present, staining was confined to the cytoplasm and membrane of malignant epithelial cells (Fig. 2C). We did not observe differences in the pCrkL protein expression pattern when invasive ductal carcinomas were compared to invasive lobular carcinomas ( $49 \%$ vs. $44 \%$, respectively, $p=0.27$, Fisher's exact test). Since there was only one case of a pure ductal carcinoma in situ (DCIS), no statement can be made regarding the prevalence of pCrkL in DCIS. In the 70 cases of colon cancer analyzed, the activated form of CrkL was more common in adenocarcinomas $(67 \%)$ than in mucinous carcinomas (17\%, $\mathrm{p}=0.02$; Fisher's exact test) with expression levels ranging from weak $(+)$ to strong $(+++)$ in both subtypes. Staining was again confined to the malignant epithelium, areas of stromal tissue remained pCrkL-negative (Fig. 2E), and the overall protein expression was significantly more common than in corresponding normal tissues ( $\mathrm{p}=0.03$; Fisher's exact test). In lung carcinomas, pCrkL staining was present in $55 \%$ of 71 biopsies, and again significantly more prevalent when compared to normal lung $(\mathrm{p}=0.00015$; Fisher's exact test). Protein staining varied considerably from weak $(+)$ in $37 \%$ and intermediate $(++)$ in $14 \%$ to strong $(+++)$ in $4 \%$, and immunodetectable pCrkL was usually epithelium-associated
(Fig. 2G). In lung cancer, the cytoplasmatic expression of activated CrkL was more common in adenocarcinomas (72\%) than in squamous carcinomas $(44 \%, \mathrm{p}=0.023$; Fisher's exact test), and there were too few cases to investigate the expression in mucinous and bronchoalveolar subtypes.

In lymphomas, pCrkL was detected in $80 \%$ of the 50 cases investigated, and no difference in the expression pattern was observed when Hodgkin's lymphomas were compared with non-Hodgkin's lymphomas. The majority of pCrkLpositive tumors exhibited intermediate $(48 \%)$ to strong $(15 \%)$ staining (Fig. 2I), and tumoral expression was significantly more common than expression in normal lymphatic tissue ( $\mathrm{p}=0.012$; Fisher's exact test). A comparable pattern was seen for skin tumors, which displayed epithelial pCrkL in $76 \%$ of 89 cases analyzed by immunohistochemistry (Fig. 2K). Intermediate $(++)$ to strong $(+++)$ staining was again observed in the majority (i.e. $58 \%$ ) of positive cases, and overall pCrkL expression was significantly more common than in corresponding normal skin ( $\mathrm{p}=0.02$; Fisher's exact test). We did not observe significant differences in pCrkL expression between melanomas, basal cell cancers and squamous cell tumors (data not shown).

pCrkL protein was detected in $50 \%$ of ovarian carcinomas and thus more common than in normal ovaries $(\mathrm{p}<0.0001$; Fisher's exact test). The prevalence was comparable in all three histological subtypes analyzed (serous vs. endometroid vs. mucinous/clear cell was $51 \%, 40 \%$ and $50 \%$, respectively). The amount of immunohistochemically detected protein expression ranged from low (25\%) to intermediate $(25 \%)$ levels, and none of the tumor samples were found to express high amounts of activated CrkL (Fig. 2M).

In sharp contrast to all other malignancies investigated, prostate cancer samples expressed immunohistochemically detectable pCrkL in only $14 \%$ of cases, which was significantly less than that found in non-malignant prostate tissue $(\mathrm{p}<0.0001)$. Of the 73 samples of adenocarcinoma investigated, only 6 showed low (8\%) and $4(5 \%)$ intermediate expression levels and protein expression was again localized in the tumor epithelium (Fig. 2O). We did not observe a single case with high levels of protein expression in this tumor entity.

$p C r k L$ expression in response to imatinib. To investigate the value of $\mathrm{pCrkL}$ in the identification of tumors that are potentially sensitive to TKI treatment, we used the CMLderived lymphoblast cell line K256 as an in vitro model and analyzed the effect of imatinib on the phosphorylation (i.e. activation) state of CrkL. Upon semi-confluency, cells were subjected to either $500 \mathrm{nM}$ imatinib mesylate or medium alone. Immunocytochemistry for pCrkL revealed that in the absence of imatinib, K256 cells expressed clearly detectable pCrkL protein (Fig. 3A). However, when the same cells were incubated with imatinib, pCrkL expression was almost completely abrogated (Fig. 3B), thus demonstrating that imatinib is able to prevent downstream activation of CrkL by inhibiting TK-dependent trans-phosphorylation.

\section{Discussion}

In recent years, there has been tremendous interest in targeted therapies, and several studies involving the use of 
synthetic TKIs have been initiated. However, with the notable exception of imatinib and erlotinib (Tarceva $\left.{ }^{\circledR}\right)$, the results of many studies have been disappointing. Several reasons can be attributed to the apparent failures, but one of the main obstacles might be the difficulty in identifying appropriate targets. Unlike in CML where translocation of bcrabl results in the activation of a specific and pathognomic tyrosine kinase, and in GIST where a mutation of c-kit causes the constitutive activation of c-Kit, the mere overexpression of a TK or its stimulating ligand may not be sufficient for effective TKI monotherapy. It is important to understand that the highly selective TKIs can sometimes only block one of several pathways, and a potential clinical benefit is found in the combinatory use of multiple TKIs or simultaneous application together with endocrine or cytotoxic drugs.

By investigating a potential surrogate parameter of therapeutic TK inhibition that is selectively up-regulated in malignant tumors, we have identified pCrkL as a key phosphoprotein involved in the signal transduction cascade of those tyrosine kinase proteins that can be blocked by TKIs in vitro. Although comparatively little is still known about receptor-mediated activation of CrkL, existing data strongly suggest a major role of PDGF-receptor (PDGF-R), c-Kit, and $\mathrm{c}-\mathrm{Abl}$ in the transphosphorylation of $\mathrm{CrkL}$ and related factors: there is a clear link between PDGF-R activation and CrkL phosphorylation; and within PDGF-R, phosphorylated Tyr-762 serves as a docking site for downstream signal transduction molecules such as CrkL and results in their consecutive activation (14). In a similar fashion, stem cell factor (SCF) binding to c-Kit, which possesses an intrinsic tyrosine kinase activity, induces rapid tyrosine auto-phosphorylation and leads to the trans-phosphorylation of several cellular proteins, including CrkL (15). Finally, CrkL can be phosphorylated by interacting with the inducible tyrosine kinase $\mathrm{c}-\mathrm{Abl}$ or the chimeric and constituitively activated Bcr-Abl fusion protein in specific forms of CML (16).

Our finding of pCrkL in normal brain tissue and the majority of astrogliomas complements a report by Sasahara et al, who observed specific immunostaining for the PDGF-B chain in neurons, principal dendrites, some axons, and probable terminals throughout the brain (17). A functional role for PDGF-B in normal brain has also been suggested by Zhang et al, who have demonstrated that in neurons and type 1 astrocytes in vitro, PDGF-BB greatly enhances protein phosphorylation, while PDGF-AA has less of an effect on protein phosphorylation (18). Furthermore, both PDGF-R and its ligand PDGF are highly expressed in astrocytomas and thought to result in considerable PDGF-R stimulation via auto- and paracrine mechanisms $(19,20)$. It is thus quite possible that the relatively high percentage of pCrkL-positive cells in normal and malignant cells is due to PDGF-R activation. Nevertheless, c-Kit and its ligand SCF could also be involved in the neuronal CrkL activation since both have a striking regional distribution and can be detected in the central nervous system, particularly in the cerebellum and hippocampus (21).

There are several reports on the expression of a functional PDGF/PDGF-R system in human breast malignancies, and most publications agree on the epithelium being the source of local PDGF production rather than surrounding breast stroma. PDGF-R expression, on the other hand, is mainly confined to adjacent stromal cells, which would indicate the existence of a paracrine (and possibly an autocrine) receptor stimulation (22). In normal breast tissue and fibroadenomas of the breast, the expression of PDGF and its receptor is considerably less common, which would also fit our observation that none of the five normal breast tissues expressed activated CrkL (23). Interestingly, Ulivi et al found both c-Kit and SCF to be highly expressed in normal breast tissue, but observed a progressive loss of expression during the process of malignant transformation (24). A similar expression pattern has also been described in colon cancer where c-Kit is rarely expressed in contrast to normal tissue (25). In colon cancer, the expression of c-Kit and SCF has been demonstrated in several colon cancer cell lines, and the aberrant activation of c-Kit appears to enhance their invasive potential while simultaneously protecting them against apoptosis $(26,27)$. Our observation of pCrkL protein expression in the majority of lung cancer samples, but in none of the normal tissues is also concordant with findings by Tsuura et al, who detected c-Kit protein in malignant but not in normal lung epithelium, and findings by Antoniades et al, who detected both PDGF and PDGF-R in malignant epithelium of lung cancer biopsies, while samples from normal lung tissue expressed neither ligand nor receptor $(28,29)$.

It has also been demonstrated that imatinib and SU11248 are both able to inhibit PDGF-R and c-Kit signaling in a preclinical model, thereby supporting their therapeutic potential (30). In melanomas and ovarian cancer, PDGF-R, c-Kit and/or c-Abl have all been detected in the majority of cases, and could be responsible for the activation of CrkL seen in these tumors $(31,32)$. Interestingly, in prostate cancer, the relatively sparse expression of pCrkL $(15 \%)$ in our patient cohort matches the lack of a functional PDGF/PDGF-R and SCF/c-Kit system expression in most of the tumors $(33,34)$. Indeed, the only medical trial investigating the efficacy of imatinib in androgenindependent prostate cancer was stopped early because of a lack of clinical activity, although there is no information about the level of CrkL activity or PDGF-R and c-Kit expression (35). We therefore suggest that pCrkL, which is selectively upregulated in several malignant tumors, could be used as a surrogate marker for the activity of TKs such as PDGF-R, $\mathrm{c}-\mathrm{Kit}$ and $\mathrm{c}-\mathrm{Abl}$, and might thus potentially be useful in monitoring the effect of therapeutic TK inhibition. Clinical studies are warranted to confirm the predictive value of pCrkL.

\section{Acknowledgements}

We thank Dr Ernst Rücklinger, Statistical Analyses Methodical Consulting, Vienna, Austria, and Niki Paucz and Kerstin Pischinger for technical assistance. This study was supported by grants from the 'Jubiläumsfonds der Österreichischen Nationalbank,' the 'Medizinisch Wissenschaftlicher Fonds des Bürgermeisters der Stadt Wien,' and Novartis Austria $\mathrm{GmbH}$.

\section{References}

1. Mayer BJ, Hamaguchi $M$ and Hanafusa $H$ : A novel viral oncogene with structural similarity to phospholipase C. Nature 332: 272-275, 1998. 
2. Prosser S, Sorokina E, Pratt P and Sorokin A: CrkIII: a novel and biologically distinct member of the Crk family of adaptor proteins. Oncogene 22: 4799-4806, 2003.

3. Pendergast AM, Quilliam LA, Cripe LD, Bassing CH, Dai Z, Li N, Batzer A, Rabun KM, Der CJ, Schlessinger J, et al: BcrAbl-induced oncogenesis is mediated by direct interaction with the SH2 domain of the GRB-2 adaptor protein. Cell 75: 175-185, 1993.

4. Ten Hoeve J, Morris C, Heisterkamp N and Groffen J: Isolation and chromosomal localization of CRKL, a human crk-like gene. Oncogene 8: 2469-2474, 1993.

5. Feller SM: Crk family adaptors-signalling complex formation and biological roles. Oncogene 20: 6348-6371, 2001.

6. Oda T, Heaney C, Hagopian JR, Okuda K, Griffin JD and Druker BJ: Crkl is the major tyrosine-phosphorylated protein in neutrophils from patients with chronic myelogenous leukemia. J Biol Chem 269: 22925-22928, 1994.

7. Rhodes J, York RD, Tara D, Tajinda K and Druker BJ: CrkL functions as a nuclear adaptor and transcriptional activator in Bcr-Abl-expressing cells. Exp Hematol 28: 305-310, 2000.

8. Lugo TG, Pendergast AM, Muller AJ and Witte ON: Tyrosine kinase activity and transformation potency of bcr-abl oncogene products. Science 247: 1079-1082, 1990.

9. Uemura N, Salgia R, Ewaniuk DS, Little MT and Griffin JD: Involvement of the adapter protein CRKL in integrin-mediated adhesion. Oncogene 18: 3343-3353, 1999.

10. Lamorte L, Royal I, Naujokas M and Park M: Crk adapter proteins promote an epithelial-mesenchymal-like transition and are required for HGF-mediated cell spreading and breakdown of epithelial adherens junctions. Mol Biol Cell 13: 1449-1461, 2002.

11. Klemke RL, Leng J, Molander R, Brooks PC, Vuori K and Cheresh DA: CAS/Crk coupling serves as a 'molecular switch' for induction of cell migration. J Cell Biol 140: 961-972, 1998.

12. Stupack DG, Cho SY and Klemke RL: Molecular signaling mechanisms of cell migration and invasion. Immunol Res 21: 83-88, 2000

13. Remmele W and Schicketanz KH: Immunohistochemical determination of estrogen and progesterone receptor content in human breast cancer. Computer-assisted image analysis (QIC score) vs. subjective grading (IRS). Pathol Res Pract 189: 862-866, 1993.

14. Yokote K, Hellman U, Ekman S, Saito Y, Ronnstrand L, Saito Y, Heldin CH and Mori S: Identification of Tyr-762 in the plateletderived growth factor alpha-receptor as the binding site for Crk proteins. Oncogene 16: 1229-1239, 1998.

15. Sattler M, Salgia R, Shrikhande G, Verma S, Pisick E, Prasad KV and Griffin JD: Steel factor induces tyrosine phosphorylation of CRKL and binding of CRKL to a complex containing c-kit, phosphatidylinositol 3-kinase, and p120(CBL). J Biol Chem 272: 10248-10253, 1997.

16. Nishihara T, Miura Y, Tohyama Y, Mizutani C, Hishita T, Ichiyama S, Uchiyama T and Tohyama K: Effects of the tyrosine kinase inhibitor imatinib mesylate on a Bcr-Abl-positive cell line: suppression of autonomous cell growth but no effect on decreased adhesive property and morphological changes. Int $\mathbf{J}$ Hematol 78: 233-240, 2003.

17. Sasahara M, Fries JW, Raines EW, Gown AM, Westrum LE, Frosch MP, Bonthron DT, Ross R and Collins T: PDGF B-chain in neurons of the central nervous system, posterior pituitary, and in a transgenic model. Cell 64: 217-227, 1991.

18. Zhang FX and Hutchins JB: Protein phosphorylation in response to PDGF stimulation in cultured neurons and astrocytes. Brain Res Dev Brain Res 99: 216-225, 1997.

19. Lokker NA, Sullivan CM, Hollenbach SJ, Israel MA and Giese NA: Platelet-derived growth factor (PDGF) autocrine signaling regulates survival and mitogenic pathways in glioblastoma cells: evidence that the novel PDGF-C and PDGFD ligands may play a role in the development of brain tumors. Cancer Res 62: 3729-3735, 2002.

20. Takeuchi H, Kanzawa T, Kondo Y and Kondo S: Inhibition of platelet-derived growth factor signalling induces autophagy in malignant glioma cells. Br J Cancer 90: 1069-1075, 2004.
21. Lammie A, Drobnjak M, Gerald W, Saad A, Cote R and Cordon-Cardo C: Expression of c-kit and kit ligand proteins in normal human tissues J Histochem Cytochem 42: 1417-1425, 1994.

22. Bhardwaj B, Klassen J, Cossette N, Sterns E, Tuck A, Deeley R, Sengupta S and Elliott B: Localization of platelet-derived growth factor beta receptor expression in the periepithelial stroma of human breast carcinoma. Clin Cancer Res 2: 773-782, 1996.

23. Feakins RM, Wells CA, Young KA and Sheaff MT: Plateletderived growth factor expression in phyllodes tumors and fibroadenomas of the breast. Hum Pathol 31: 1214-1222, 2000.

24. Ulivi P, Zoli W, Medri L, Amadori D, Saragoni L, Barbanti F, Calistri D and Silvestrini R: c-kit and SCF expression in normal and tumor breast tissue. Breast Cancer Res Treat 83: 33-42, 2004.

25. Sammarco I, Capurso G, Coppola L, Bonifazi AP, Cassetta S, Delle Fave G, Carrara A, Grassi GB, Rossi P, Sette C and Geremia R: Expression of the proto-oncogene c-KIT in normal and tumor tissues from colorectal carcinoma patients. Int $\mathbf{J}$ Colorectal Dis 19: 545-553, 2004.

26. Toyota M, Hinoda Y, Takaoka A, Makiguchi Y, Takahashi T, Itoh $\mathrm{F}$, Imai $\mathrm{K}$ and Yachi $\mathrm{A}$ : Expression of c-kit and kit ligand in human colon carcinoma cells. Tumour Biol 14: 295-302, 1993.

27. Bellone G, Carbone A, Sibona N, Bosco O, Tibaudi D, Smirne C, Martone T, Gramigni C, Camandona M, Emanuelli G and Rodeck U: Aberrant activation of c-kit protects colon carcinoma cells against apoptosis and enhances their invasive potential. Cancer Res 61: 2200-2206, 2001.

28. Tsuura Y, Hiraki H, Watanabe K, Igarashi S, Shimamura K, Fukuda T, Suzuki T and Seito T: Preferential localization of c-kit product in tissue mast cells, basal cells of skin, epithelial cells of breast, small cell lung carcinoma and seminoma/ dysgerminoma in human: immunohistochemical study on formalin-fixed, paraffin-embedded tissues. Virchows Arch 424: 135-134, 1994.

29. Antoniades HN, Galanopoulos T, Neville-Golden J and O'Hara CJ: Malignant epithelial cells in primary human lung carcinomas coexpress in vivo platelet-derived growth factor (PDGF) and PDGF receptor mRNAs and their protein products. Proc Natl Acad Sci USA 89: 3942-3946, 1992.

30. Abrams TJ, Lee LB, Murray LJ, Pryer NK and Cherrington JM: SU11248 inhibits KIT and platelet-derived growth factor receptor beta in preclinical models of human small cell lung cancer. Mol Cancer Ther 2: 471-478, 2003.

31. McGary EC, Onn A, Mills L, Heimberger A, Eton O, Thomas GW, Shtivelband M and Bar-Eli M: Imatinib mesylate inhibits platelet-derived growth factor receptor phosphorylation of melanoma cells but does not affect tumorigenicity in vivo. J Invest Dermatol 122: 400-405, 2004.

32. Schmandt RE, Broaddus R, Lu KH, Shvartsman H, Thornton A, Malpica A, Sun C, Bodurka DC and Gershenson DM: Expression of c-ABL, c-KIT, and platelet-derived growth factor receptor-beta in ovarian serous carcinoma and normal ovarian surface epithelium. Cancer 98: 758-764, 2003.

33. Simak R, Capodieci P, Cohen DW, Fair WR, Scher H, Melamed J, Drobnjak M, Heston WD, Stix U, Steiner G and Cordon-Cardo C: Expression of c-kit and kit-ligand in benign and malignant prostatic tissues. Histol Histopathol 15: 365-374, 2000.

34. Fudge K, Wang CY and Stearns ME: Immunohistochemistry analysis of platelet-derived growth factor A and B chains and platelet-derived growth factor alpha and beta receptor expression in benign prostatic hyperplasias and Gleason-graded human prostate adenocarcinomas. Mod Pathol 7: 549-554, 1994.

35. Tiffany NM, Wersinger EM, Garzotto $M$ and Beer TM: Imatinib mesylate and zoledronic acid in androgen-independent prostate cancer. Urology 63: 934-939, 2004. 